\title{
Estimation of the Parameters of Compound Weibull Distribution
}

\author{
Dr. Neamat Qutb ${ }^{1}$, Elham Rajhi ${ }^{2}$ \\ ${ }^{1,2}$ (Department Of Statistics, Faculty Of Science/ King Abdulaziz University, Saudi Arabia.) \\ ${ }_{2}^{2}$ (Basic Sciences Department, AL-Farabi college, Jeddah, Saudi Arabia)
}

\begin{abstract}
This paper explores the potential of the Compound Weibull Distribution $(\mathrm{CW})$ in theory and practice. Statistical lifetime distributions are commonly used in various fields for modeling data sets where the CW distribution is the most applicable. This distribution is presented with its properties and graphical representations. Some special cases and related distributions to the $C W$ distribution are derived. Moments $(M)$, maximum likelihood (ML) and Bayesian - based on informative prior - methods are used to estimate the unknown parameters of the distribution. Markov Chain Monte Carlo (MCMC) technique is used to compute the Bayesian estimates with different prior distributions based on squared and absolute error loss functions. The simulation is performed to investigate and compare between the estimators with different sizes and one set of the parameter's values. In the sense of the mean squared error (MSE), the results showed that case 1 of Bayesian method, with gamma prior distribution and under squared error loss function (B1sq) is the best estimation method. A real data set is used to compare between the Weibull and the CW distributions and the results showed that the $C W$ distribution provides better fit than the Weibull distribution.
\end{abstract}

Keywords: Compound Weibull Distribution, maximum likelihood, Bayesian estimation, MCMC, error loss functions

\section{Introduction}

Compound distributions gained their importance from the fact that natural factors have compound effects, as in the medical, social and logical experiments. On the other hand, many relations are being characterized as being contagious, such as bacterization. In such cases, a simple distribution is assigned to describe each factor in the complex phenomenon (or each stage in the contagious data) and combining them in one distribution called compound distribution.

CW distribution was first introduced by [1], where she compounded Weibull distribution with gamma distribution, and by means of suitable transformations she reduced it to the $\mathrm{F}$ and beta distributions. [2] derived theoretical expressions for low flow distribution by $\mathrm{CW}$ distribution and used L-moments and probability weighted moments methods to estimate its parameters. [3] studied various properties of the $\mathrm{CW}$ distribution and used the ML method for estimating its parameters. [4] derived new recurrence relations satisfied by the product and single moments of the progressively type two right censored order statistics from CW distribution and its truncated form, they obtained the ML estimators and the best linear unbiased estimators of the scale and location parameters and used a simulation method to make comparison between the MSE of them.

If $\mathrm{X}$ is a random variable from known distribution and one of its parameters $\theta$ is considered as a random variable with a specified distribution then, we can say that $X$ has a compound distribution [5]. The general form of the compound density function is

$$
f(x)=\int_{\theta} f(x \mid \theta) f(\theta) d \theta,
$$

where $f(x \mid \theta)$ is a conditional density function depending on the parameter $\theta$, which is considered as a random variable with density function $f(\theta)$ that will be called the compounder distribution. The variable that has distribution function (1) will be symbolized by $\mathrm{X} \wedge \theta$ and will be called a compound of the variable $\mathrm{X}$ with respect to the compounding $\theta$. Relation (1) will be symbolized as

$$
f(x) \equiv f(x \mid \theta)_{\hat{\theta}} f(\theta),
$$

where the symbol $\Lambda$ is the compounding operator [6].

This paper is organized as follows: Section 1, introduction to CW distribution. Section 2 presents properties of the $\mathrm{CW}$ distribution, special cases and related distributions of the $\mathrm{CW}$ distribution are derived, estimation of the unknown parameters of the distribution using M, ML and Bayesian methods, a simulation study is performed to investigate these estimation methods and a real data set is used to compare between Weibull and CW distributions. The conclusions are shown in Section 3. 


\section{From Weibull to Compound Weibull Distribution}

In this section, the functions of the $\mathrm{CW}$ distribution are presented with graphical representation; probability density function (PDF), cumulative distribution function (CDF), reliability function (RF) and hazard rate function (HRF). Some of the important properties of the $\mathrm{CW}$ distribution are introduced; $\mathrm{r}^{\text {th }}$ moment, mean, variance, skewness, kurtosis, mode and median.

[1] derived the $\mathrm{CW}$ distribution by assuming a conditional random variable $\mathrm{X}$ follow the Weibull distribution, and its scale parameter follow a gamma distribution. The resulting unconditional PDF is called the CW distribution.

Let the conditional random variable $\mathrm{X}$ follow the Weibull distribution with two parameters whose PDF is given by

$$
f(x \mid \alpha, \beta)=\alpha \beta x^{\alpha-1} \exp \left(-\beta x^{\alpha}\right), \quad x \geq 0, \alpha \text { and } \beta>0,
$$

where $\alpha$ is a shape parameter and $\beta$ is a scale parameter.

With CDF:

$$
F(x ; \alpha, \beta)=1-\exp \left(-\beta x^{\alpha}\right) .
$$

The RF and HRF of Weibull distribution are given respectively by

$$
\begin{gathered}
R(x ; \alpha, \beta)=\exp \left(-\beta x^{\alpha}\right), \\
h(x ; \alpha, \beta)=\alpha \beta x^{\alpha-1} .
\end{gathered}
$$

From equation (6) and Fig. 1 it is clear that, the HRF of Weibull distribution is increasing for $\alpha>1$, constant for $\alpha=1$ and decreasing for $\alpha<1$. This property of having a flexible hazard function, which can take a decreasing, constant or increasing shape over time, makes the Weibull distribution applicable in many fields. It is widely used distribution in reliability and lifetime data analysis such as: reliability engineering, material science, physics, chemistry, meteorology and hydrology, medicine, economics and business, biology and forestry and many other areas [7].

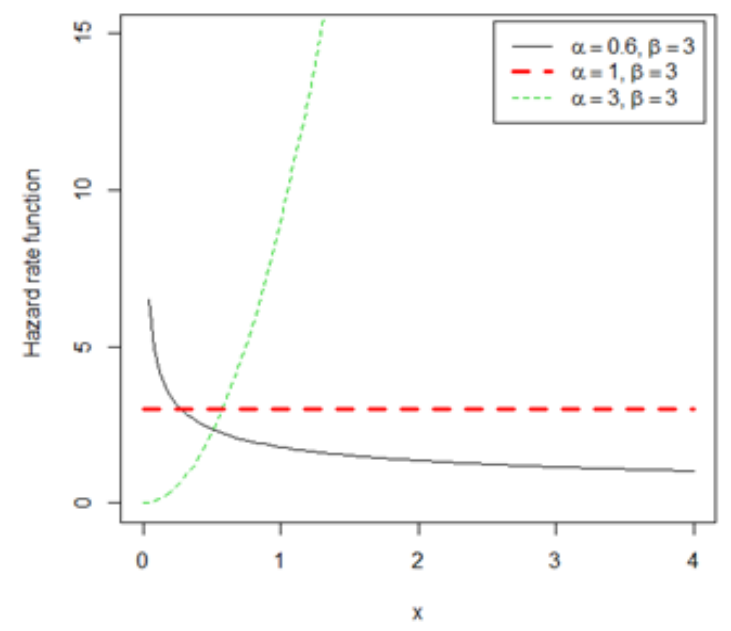

Figure 1: The HRF of the Weibull distribution

Let the scale parameter $\beta$ of the Weibull distribution with PDF given in (3) follow a gamma distribution with parameters $\delta$ and $\gamma$, then the PDF of $\beta$ can be written as:

$$
f(\beta ; \gamma, \delta)=\frac{\delta^{\gamma} \beta^{\gamma-1} e^{-\delta \beta}}{\Gamma_{(\gamma)}}, \beta \geq 0, \gamma \text { and } \delta>0 .
$$

Now, the Weibull $\bigwedge_{\beta}$ Gamma can be obtained as:

$$
f(x ; \alpha, \gamma, \delta)=\frac{\alpha \gamma \delta^{\gamma} x^{\alpha-1}}{\left(x^{\alpha}+\delta\right)^{\gamma+1}}, \quad x \geq 0, \alpha, \gamma \text { and } \delta>0,
$$

with shape parameters $\alpha$ and $\gamma$, and scale parameter $\delta$. The above PDF (8) is the PDF of the CW distribution. Fig. 2 represents the PDF for the $\mathrm{CW}$ distribution when considering different values of its parameters. It is clear that the shape of the $\mathrm{CW}$ distribution is decreasing if $\alpha \leq 1$ and unimodal if $\alpha>1$, with right skewness.

The $\mathrm{CDF}$ of the $\mathrm{CW}$ distribution is given by

$$
F(x ; \alpha, \gamma, \delta)=1-\frac{\delta^{\gamma}}{\left(x^{\alpha}+\delta\right)^{\gamma}} .
$$

Fig. 3 represents the $\mathrm{CDF}$ for the $\mathrm{CW}$ distribution when its parameters take different values. 


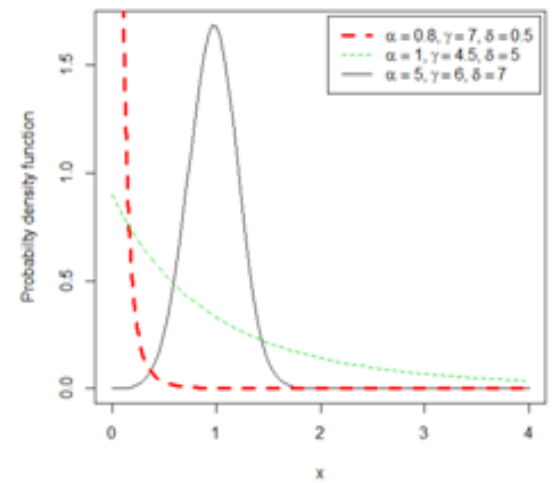

Figure 2: The PDF of the $\mathrm{CW}$ distribution

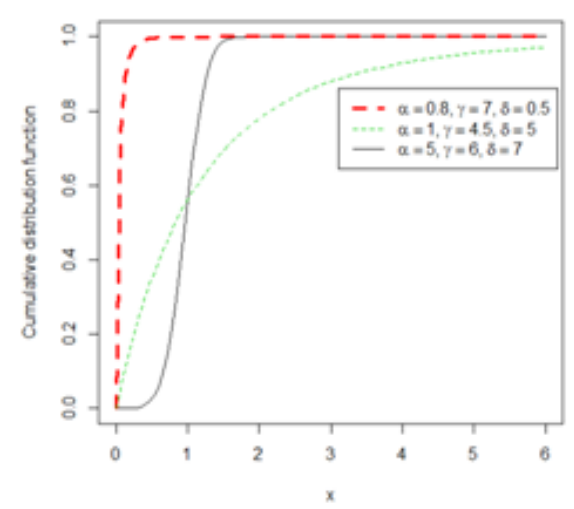

Figure 3: The $\mathrm{CDF}$ of the $\mathrm{CW}$ distribution

The quantile function corresponding to the distribution in equation (9) is given by

$$
x(F)=\delta^{1 / \alpha}\left[(1-F)^{-1 / \gamma}-1\right]^{1 / \alpha} .
$$

The RF and HRF for the $\mathrm{CW}$ distribution are given respectively by

$$
\begin{aligned}
& R(x ; \alpha, \gamma, \delta)=\frac{\delta^{\gamma}}{\left(\delta+x^{\alpha}\right)^{\gamma}}, \\
& h(x ; \alpha, \gamma, \delta)=\frac{\alpha \gamma x^{\alpha-1}}{\delta+x^{\alpha}} .
\end{aligned}
$$

Fig. 4 represents the HRF and it is clear that:

(1) $h(x ; \alpha, \beta)$ is a decreasing function of $\mathrm{x}$ at $\alpha<1$.

(2) $h(x ; \alpha, \beta)$ is approximately constant function of $\mathrm{x}$ at $\alpha=1$.

(3) $h(x ; \alpha, \beta)$ is a unimodal shaped when $\alpha>1$.

This property of having a flexible hazard function, which can take a decreasing, approximately constant or unimodal shape over time, makes the $\mathrm{CW}$ distribution as the Weibull distribution, applicable in many fields.

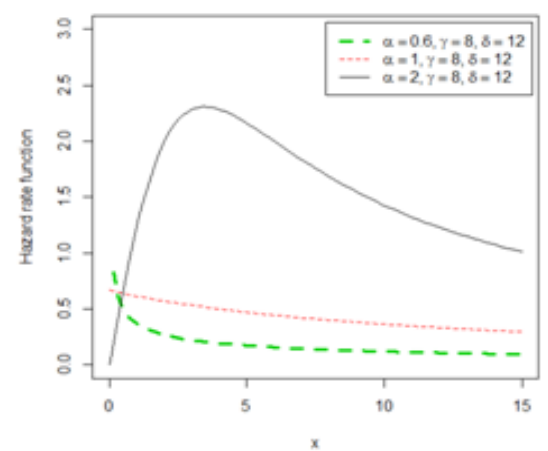

Figure 4: The HRF of the $\mathrm{CW}$ distribution 
The $\mathrm{r}^{\text {th }}$ moment of the $\mathrm{CW}$ distribution is given by

$$
\mu_{r}^{\prime}=\gamma \delta^{r / \alpha} B\left(\gamma-\frac{r}{\alpha}, \frac{r}{\alpha}+1\right)
$$

Provided that $\alpha \gamma>r$. The mean and variance of the $\mathrm{CW}$ distribution are given respectively by

$$
\begin{gathered}
\mu=\frac{\delta^{1 / \alpha} \Gamma\left(\gamma-\frac{1}{\alpha}\right) \Gamma\left(\frac{1}{\alpha}+1\right)}{\Gamma(\gamma)} \\
\sigma^{2}=\frac{\delta^{2 / \alpha}}{\Gamma_{(\gamma)}}\left[\Gamma\left(\gamma-\frac{2}{\alpha}\right) \Gamma\left(\frac{2}{\alpha}+1\right)-\frac{1}{\Gamma_{(\gamma)}} \Gamma^{2}\left(\gamma-\frac{1}{\alpha}\right) \Gamma^{2}\left(\frac{1}{\alpha}+1\right)\right] .
\end{gathered}
$$

The skewness and kurtosis are given respectively by

$$
\begin{gathered}
\propto_{3}=\frac{[\Gamma(\gamma)]^{1 / 2} \Gamma\left(\gamma-\frac{3}{\alpha}\right) \Gamma\left(\frac{3}{\alpha}+1\right)}{\left[\Gamma\left(\gamma-\frac{2}{\alpha}\right)\right]^{3 / 2}\left[\Gamma\left(\frac{2}{\alpha}+1\right)\right]^{3 / 2}}, \\
\propto_{4}=\frac{\Gamma(\gamma) \Gamma\left(\gamma-\frac{4}{\alpha}\right) \Gamma\left(\frac{4}{\alpha}+1\right)}{\Gamma^{2}\left(\gamma-\frac{2}{\alpha}\right) \Gamma^{2}\left(\frac{2}{\alpha}+1\right)} .
\end{gathered}
$$

The mode of the $\mathrm{CW}$ distribution is at the point

$$
x=\sqrt[\alpha]{\frac{\alpha-1}{\delta^{-1}(\alpha \gamma+1)}}
$$

this mode exists only for $\alpha>1$, and the median can be obtained as

$$
m=\sqrt[\alpha]{\frac{\sqrt[\gamma]{2}-1}{\delta^{-1}}} .
$$

\subsection{Special cases and related distributions}

In this subsection, special cases and related distributions to the $\mathrm{CW}$ distribution are presented. Burr type $\operatorname{XII}(\alpha, \gamma)$, compound exponential-gamma $(\gamma, \delta)$, compound Weibull-exponential $\left(\alpha, \delta^{-1}\right)$ and compound exponential-exponential $(\delta)$ distributions are all special cases of the $\mathrm{CW}$ distribution [1]. The $\mathrm{CW}$ distribution is related through variable transformations to a wide range of known distributions such as type $\mathrm{I}$ beta $(\gamma, 1)$, type $\Pi$

\begin{tabular}{|c|c|c|c|}
\hline Transformation & Distribution & PDF & Range \\
\hline$Y_{1}=\frac{\delta}{\left(x^{\alpha}+\delta\right)}$ & Type I beta $(\gamma, 1)$ & $\gamma y_{1}^{\gamma-1}$ & $\begin{array}{c}0 \leq \mathrm{y}_{1} \leq 1 \\
\gamma>0\end{array}$ \\
\hline$Y_{2}=\frac{x^{\alpha}}{\delta}$ & Type II beta $(1, \gamma)$ & $\frac{\gamma}{\left(1+y_{2}\right)^{\gamma+1}}$ & $\begin{aligned} y_{2} & >0 \\
\gamma & >0\end{aligned}$ \\
\hline$Y_{3}=\frac{\gamma x^{\alpha}}{\delta}$ & $\mathrm{F}(2,2 \gamma)$ & $\frac{1}{\left(1+\frac{y_{3}}{\gamma}\right)^{\gamma+1}}$ & $\begin{array}{c}y_{3}>0 \\
\gamma>0\end{array}$ \\
\hline$Y_{4}=x^{\alpha}$ & Pareto2(a,c) & $a c^{a}\left(y_{4}+c\right)^{-(a+1)}$ & $\begin{array}{l}y_{4}>0 \\
a, c>0\end{array}$ \\
\hline$Y_{5}=\ln \left(1+\delta^{-1} x^{\alpha}\right)$ & exponential $(\gamma)$ & $\gamma e^{-\gamma y_{5}}$ & $\begin{aligned} y_{5} & >0 \\
\gamma & >0\end{aligned}$ \\
\hline
\end{tabular}
beta $(1, \gamma), \mathrm{F}(2,2 \gamma)$ distributions [1], Pareto type $\Pi(\mathrm{a}, \mathrm{c})$, exponential $(\gamma)$ and $\operatorname{Burr}$ type $\mathrm{XII}(\alpha, \gamma)$ distributions [3]. Some other special cases and related distributions to the CW distribution are derived. Tables 1 and 2 summarized the special cases and related distribution to the $\mathrm{CW}$ distribution.

Table 1: Summary of special cases of the CW distribution

\begin{tabular}{|c|c|c|c|}
\hline $\begin{array}{c}\text { Value of the } \\
\text { parameters }\end{array}$ & Distribution & PDF & Range \\
\hline$\delta=1$ & Burr type XII $(\alpha, \gamma)$ & $\frac{\alpha \gamma x^{\alpha-1}}{\left(1+x^{\alpha}\right)^{\gamma+1}}$ & $\gamma>0, \alpha, \gamma>0$ \\
\hline$\alpha=1$ & $\begin{array}{c}\text { Compound exponential-gamma } \\
(\gamma, \delta)\end{array}$ & $\delta^{\gamma}(x+\delta)^{-(\gamma+1)}$ & $x>0, \gamma, \delta>0$ \\
\hline$\gamma=1$ & $\begin{array}{c}\text { Compound Weibull- } \\
\text { exponential }\left(\alpha, \delta^{-1}\right)\end{array}$ & $\delta^{-1} \alpha x^{\alpha-1}\left(1+\delta^{-1} x^{\alpha}\right)^{-2}$ & $x>0, \alpha>0$ \\
\hline$\alpha=\gamma=1$ & $\begin{array}{c}\text { Compound exponential- } \\
\text { exponential }(\delta)\end{array}$ & $\frac{\delta(x+\delta)^{-2}}{\gamma}$ & $x>0, \delta>0$ \\
\hline$\alpha=\delta=1$ & $\begin{array}{c}\text { Type } \Pi \text { beta }(1, \gamma) \\
(1+x)^{\gamma+1}\end{array}$ & $x>0, \gamma>0$ \\
\hline
\end{tabular}

Table 2: Summary of transformations applied to the CW distribution and resulting distributions 
Table 2 Continued: Summary of transformations applied to the CW distribution and resulting distributions

\begin{tabular}{|c|c|c|c|}
\hline Transformation & Distribution & PDF & Range \\
\hline$Y_{6}=\delta^{-1 / \alpha} x$ & Burr type XII $(\alpha, \gamma)$ & $\frac{\alpha \gamma y_{6}{ }^{\alpha-1}}{\left(1+y_{6}{ }^{\alpha}\right)^{\gamma+1}}$ & $\begin{array}{l}y_{6}>0 \\
\alpha, \gamma>0\end{array}$ \\
\hline $\begin{array}{c}Y_{7}= \\
{\left[\ln \left(1+\delta^{-1} x^{\alpha}\right)\right]^{1 / \alpha}}\end{array}$ & Weibull $(\alpha, \gamma)$ & $\alpha \gamma y_{7}^{\alpha-1} \exp \left(-\gamma y_{7}^{\alpha}\right)$ & $\begin{array}{l}y_{7} \geq 0 \\
\alpha, \gamma>0\end{array}$ \\
\hline $\begin{array}{c}Y_{8}= \\
{\left[\ln \left(1+\delta^{-1} x^{\alpha}\right)\right]^{1 / 2}}\end{array}$ & Rayleigh $(\gamma)$ & $2 \gamma y_{8} \exp \left(-\gamma y_{8}^{2}\right)$ & $\begin{array}{c}y_{8}>0 \\
\gamma>0\end{array}$ \\
\hline $\begin{array}{c}Y_{9}= \\
\left(1+\delta^{-1} x^{\alpha}\right)\end{array}$ & $\operatorname{Pareto}(\gamma, 1)$ & $\gamma y_{9}^{-(\gamma+1)}$ & $\begin{array}{c}y_{9}>1 \\
\gamma>0\end{array}$ \\
\hline $\begin{array}{c}Y_{10}= \\
\frac{1}{\alpha} \ln \left(1+\alpha x^{\alpha}\right)\end{array}$ & $\begin{array}{c}\text { Compound } \\
\operatorname{Gompertz}(\alpha, \gamma, \delta)\end{array}$ & $\frac{\gamma}{\delta} \exp \left(\alpha y_{10}\right)\left[1+\frac{\left[\exp \left(\alpha y_{10}\right)-1\right]}{\delta \alpha}\right]^{-(\gamma+1)}$ & $\begin{array}{l}y_{10}>0 \\
\alpha, \gamma, \delta>0\end{array}$ \\
\hline $\begin{array}{c}Y_{11}= \\
\ln \left[\ln \left(1+\frac{x^{\alpha}}{\delta}\right)^{\gamma}\right]^{-1 / \gamma}\end{array}$ & $\begin{array}{l}\text { Extreme } \\
\text { Value }(\gamma)\end{array}$ & $\gamma \exp \left[-\gamma y_{11}-\exp \left(-\gamma y_{11}\right)\right]$ & $\begin{array}{c}-\infty<y_{11}<\infty, \\
\gamma>0\end{array}$ \\
\hline
\end{tabular}

\subsection{Estimation methods}

In this subsection, M, ML and Bayesian methods is used to estimate the unknown parameters $\underline{\theta}=(\alpha, \gamma, \delta)$ of the $\mathrm{CW}$ distribution. Bayesian method is conducted using informative prior distribution and different prior distributions is used. The Bayes estimates under squared and absolute error loss functions is obtained.

\subsubsection{Parameters estimation of the compound Weibull distribution using the method of moments}

Let $\underline{X}=\left(X_{1}, X_{2}, \ldots \ldots, X_{n}\right)$ be a random sample of size $\mathrm{n}$ from $\mathrm{CW}$ distribution, to apply the method of M for estimating $\underline{\theta}=(\alpha, \gamma, \delta)$, we need to equate the population $\mathrm{M}$ to the corresponding sample $\mathrm{M}$ (20) and then, solve the three equations simultaneously for $\underline{\theta}$.

$$
\left.\begin{array}{c}
\frac{\delta^{1 / \alpha} \Gamma\left(\gamma-\frac{1}{\alpha}\right) \Gamma\left(\frac{1}{\alpha}+1\right)}{\Gamma_{\gamma}}=\bar{x} \\
\frac{\delta^{2 / \alpha} \Gamma_{\left(\gamma-\frac{2}{\alpha}\right)} \Gamma_{\left(\frac{2}{\alpha}+1\right)}}{\Gamma_{\gamma}}=\frac{1}{n} \sum_{i=1}^{n} x_{i}^{2} \\
\frac{\delta^{3 / \alpha} \Gamma_{\left(\gamma-\frac{3}{\alpha}\right)} \Gamma_{\left(\frac{3}{\alpha}+1\right)}}{\Gamma_{\gamma}}=\frac{1}{n} \sum_{i=1}^{n} x_{i}^{3}
\end{array}\right\} .
$$

Numerical calculations are required to find the solution of the system of the nonlinear equation (20).

\subsubsection{Parameters estimation of the compound Weibull distribution using the maximum \\ likelihood method}

Let $\underline{X}=\left(X_{1}, X_{2}, \ldots, X_{n}\right)$ be a random sample of size $\mathrm{n}$ from $\mathrm{CW}$ distribution. The likelihood function $L(\alpha, \gamma, \delta)$ is given by

where for $\mathrm{i}=1,2, \ldots, \mathrm{n}, w\left(x_{i} ; \alpha, \gamma, \delta\right)=1+\delta^{-1} x_{i}^{\alpha}$.

$$
L(\alpha, \gamma, \delta)=\alpha^{n} \gamma^{n} \delta^{-n} \prod_{i=1}^{n} x_{i}^{\alpha-1} \prod_{i=1}^{n}\left(w\left(x_{i} ; \alpha, \gamma, \delta\right)\right)^{-(\gamma+1)},
$$

The log-likelihood function is given by

$$
\begin{aligned}
& \log L(\alpha, \gamma, \delta)=n \log \alpha+n \log \gamma+n \log \delta^{-1}+ \\
& \quad(\alpha-1) \sum_{i=1}^{n} \log x-(\gamma+1) \sum_{i=1}^{n} \log \left(w\left(x_{i} ; \alpha, \gamma, \delta\right)\right) .
\end{aligned}
$$

Differentiating (22) with respect to the parameters $\alpha, \gamma$ and $\delta$ respectively, and equating each of the resulting equations to zero, we get

$$
\left.\begin{array}{l}
\frac{\partial \log L(\alpha, \gamma, \delta)}{\partial \alpha}=\frac{n}{\hat{\alpha}}+\sum_{i=1}^{n} \log x-(\hat{\gamma}+1) \sum_{i=1}^{n} \frac{\widehat{\delta}^{-1} x_{i}^{\widehat{\alpha}} \log x}{w\left(x_{i} ; \widehat{\alpha}, \hat{\gamma}, \widehat{\delta}\right)}=0 \\
\frac{\partial \log L(\alpha, \gamma, \delta)}{\partial \gamma}=\frac{n}{\widehat{\gamma}}-\sum_{i=1}^{n} \log \left(w\left(x_{i} ; \hat{\alpha}, \hat{\gamma}, \hat{\delta}\right)\right)=0 \\
\frac{\partial \log L(\alpha, \gamma, \delta)}{\partial \delta}=-\frac{n}{\hat{\delta}}+(\hat{\gamma}+1) \sum_{i=1}^{n} \frac{\widehat{\delta}^{-2} x_{i}^{\widehat{\alpha}}}{w\left(x_{i} ; \widehat{\alpha}, \widehat{\gamma}, \widehat{\delta}\right)}=0
\end{array}\right\} .
$$

To obtain the ML estimates of the vector of parameters, $\underline{\theta}=(\alpha, \gamma, \delta)$, numerical calculations are required to find the solution of the system of the nonlinear equation (23). 


\subsubsection{Parameters estimation of the compound Weibull distribution using Bayesian method}

Assume that the vector of unknown parameters $\underline{\theta}=(\alpha, \gamma, \delta)$ are independent, the Bayesian estimators of the parameters $\underline{\theta}$ of the $\mathrm{CW}$ distribution are obtained using informative prior. Suppose that, there are different prior distributions; case 1 in which $\alpha, \gamma$ and $\delta$ follow gamma distribution, case 2 in which $\alpha$ follows exponential distribution and $\gamma, \delta$ follow gamma distribution. The posterior distributions, Bayes estimators under squared error loss and absolute error loss functions for the parameters under each prior will be obtained.

\subsubsection{First case}

Let each of $\alpha, \gamma$ and $\delta$ follow one parameter gamma $\left(k_{i}\right)$ distribution, $i=1,2,3$ respectively, then the prior distribution for each parameters can be written as follows

$$
\left.\begin{array}{ll}
\pi_{1}(\alpha)=\frac{1}{\Gamma\left(k_{1}\right)} \alpha^{k_{1}-1} e^{-\alpha}, & \alpha \geq 0 \\
\pi_{2}(\gamma)=\frac{1}{\Gamma\left(k_{2}\right)} \gamma^{k_{2}-1} e^{-\gamma}, & \gamma \geq 0 \\
\pi_{3}(\delta)=\frac{1}{\Gamma\left(k_{3}\right)} \delta^{k_{3}-1} e^{-\delta}, & \delta \geq 0
\end{array}\right\} .
$$

The joint prior density function of the independent parameters $\alpha, \gamma$ and $\delta$ can be obtained from equation (24) as

$$
\pi_{1}(\alpha, \gamma, \delta)=\pi_{1}(\alpha) \cdot \pi_{2}(\gamma) \cdot \pi_{3}(\delta),
$$

then the joint posterior probability density function of $\alpha, \gamma$ and $\delta$ given $\underline{x}$ can be obtained from equation (21) and (25) as

$$
\begin{gathered}
\pi_{1}^{*}(\alpha, \gamma, \delta \mid \underline{x})=A \alpha^{n} \gamma^{n} \delta^{-n} \prod_{i=1}^{n} x_{i}^{\alpha-1} \prod_{i=1}^{n}\left[w\left(x_{i} ; \alpha, \gamma, \delta\right)\right]^{-(\gamma+1)}, \\
\times \pi_{1}(\alpha, \gamma, \delta)
\end{gathered},
$$

where $\mathrm{A}$ is a normalizing constant that is

$$
\begin{gathered}
A^{-1}=\int_{0}^{\infty} \int_{0}^{\infty} \int_{0}^{\infty} \alpha^{n} \gamma^{n} \delta^{-n} \prod_{i=1}^{n} x_{i}^{\alpha-1} \prod_{i=1}^{n}\left[w\left(x_{i} ; \alpha, \gamma, \delta\right)\right]^{-(\gamma+1)} \\
\times \pi_{1}(\alpha, \gamma, \delta) d \alpha d \gamma d \delta
\end{gathered}
$$

The B1sq estimators can be obtained by taking the mean of the equation (26). Case 1 of the Bayesian estimators under absolute error loss function (B1abs) can be obtained by taking the median of (26). MCMC technique is used to find the Bayesian estimates of $\underline{\theta}$ numerically.

\subsubsection{Second case}

Let $\alpha$ follow an exponential distribution with parameter $\lambda$, and each one of the two parameters $\gamma, \delta$ follow a one parameter gamma distribution with parameter $k_{i}, i=1,2$ respectively, then the prior distribution for each of the parameters can be written as follows

$$
\left.\begin{array}{ll}
\pi_{1}(\lambda)=\lambda e^{-\lambda \alpha}, & \alpha \geq 0 \\
\pi_{2}(\gamma)=\frac{1}{\Gamma\left(k_{1}\right)} \gamma^{k_{1}-1} e^{-\gamma}, & \gamma \geq 0 \\
\pi_{3}(\delta)=\frac{1}{\Gamma\left(k_{2}\right)} \delta^{k_{2}-1} e^{-\delta}, & \delta \geq 0
\end{array}\right\} .
$$

Therefore, the joint prior density function of the independent parameters $\alpha, \gamma$ and $\delta$ can be obtained from equation (28) as

$$
\pi_{2}(\alpha, \gamma, \delta)=\pi_{1}(\alpha) \cdot \pi_{2}(\gamma) \cdot \pi_{3}(\delta),
$$

then the joint posterior probability density function of $\alpha, \gamma$ and $\delta$ given $\underline{x}$ can be obtained from equation (21) and (29) as

where $\mathrm{B}$ is a normalizing constant as

$$
\times \pi_{2}(\alpha, \gamma, \delta)
$$

$$
\begin{gathered}
B^{-1}=\int_{0}^{\infty} \int_{0}^{\infty} \int_{0}^{\infty} \alpha^{n} \gamma^{n} \delta^{-n} \prod_{i=1}^{n} x_{i}^{\alpha-1} \prod_{i=1}^{n}\left[w\left(x_{i} ; \alpha, \gamma, \delta\right)\right]^{-(\gamma+1)} \\
\times \pi_{2}(\alpha, \gamma, \delta) d \alpha d \gamma d \delta
\end{gathered}
$$

Case 2 of Bayesian estimators under squared error loss function (B2sq)can be obtained by taking the mean of the equation (30). The Bayesian estimators case 2 under absolute error loss function (B2abs) can be obtained by taking the median of (30). MCMC technique is used to find the Bayesian estimates of $\underline{\theta}$ numerically. 


\subsection{Simulation study}

In this subsection, simulation study and results are presented. Simulation study have been performed to obtain the estimates presented in (2.2) subsection. This simulation was performed for different sample size $\mathrm{n}=30,50,100$. Each sample size is repeated 1000 times. For 1000 replications, the estimated MSE, bias and Variance (Var) of the estimates are calculated for each method. The results are shown in Table 3.

Table 3: $\mathrm{M}, \mathrm{ML}$ and Bayesian estimates of the parameters of the compound Weibull distribution at

$$
\underline{\theta}=(\alpha=1.5, \gamma=2.7, \delta=1.6)
$$

\begin{tabular}{|c|c|c|c|c|c|c|c|}
\hline & & $\mathrm{M}$ & ML & B1sq & B1abs & B2sq & B2abs \\
\hline $\mathrm{n}$ & \multicolumn{7}{|c|}{30} \\
\hline \multirow[t]{3}{*}{ Estimate } & $\hat{\alpha}$ & 1.7193 & 1.6830 & 1.6821 & 1.6571 & 1.7599 & 1.7307 \\
\hline & $\hat{\gamma}$ & 3.4260 & 2.2902 & 2.2176 & 2.0500 & 2.1201 & 1.9533 \\
\hline & $\hat{\delta}$ & 2.2522 & 1.2761 & 1.2465 & 1.0969 & 1.1729 & 1.0229 \\
\hline \multirow[t]{3}{*}{ Bias } & $\hat{\alpha}$ & 0.2193 & 0.1830 & 0.1821 & 0.1571 & 0.2599 & 0.2307 \\
\hline & $\hat{\gamma}$ & 0.7260 & -0.4098 & -0.4824 & -0.6500 & -0.5799 & -0.7467 \\
\hline & $\hat{\delta}$ & 0.6522 & -0.3239 & -0.3535 & -0.5031 & -0.4271 & -0.5771 \\
\hline \multirow[t]{3}{*}{ Var } & $\hat{\alpha}$ & 0.0984 & 0.0798 & 0.0661 & 0.0630 & 0.0860 & 0.0805 \\
\hline & $\hat{\gamma}$ & 0.0896 & 0.5825 & 0.1508 & 0.1398 & 0.1692 & 0.1580 \\
\hline & $\hat{\delta}$ & 0.1780 & 0.3445 & 0.0901 & 0.0849 & 0.0963 & 0.0919 \\
\hline \multirow[t]{3}{*}{ MSE } & $\hat{\alpha}$ & 0.1464 & 0.1133 & 0.0992 & 0.0877 & 0.1535 & 0.1338 \\
\hline & $\hat{\gamma}$ & 0.6168 & 0.7505 & 0.3835 & 0.5623 & 0.5055 & 0.7156 \\
\hline & $\hat{\delta}$ & 0.6034 & 0.4494 & 0.2151 & 0.3381 & 0.2787 & 0.4250 \\
\hline $\mathrm{n}$ & \multicolumn{7}{|c|}{50} \\
\hline \multirow[t]{3}{*}{ Estimate } & $\hat{\alpha}$ & 1.6257 & 1.6186 & 1.6250 & 1.6068 & 1.6710 & 1.6508 \\
\hline & $\hat{\gamma}$ & 3.5001 & 2.3527 & 2.2971 & 2.1380 & 2.2133 & 2.0567 \\
\hline & $\hat{\delta}$ & 2.3272 & 1.3290 & 1.3041 & 1.1648 & 1.2415 & 1.1022 \\
\hline \multirow[t]{3}{*}{ Bias } & $\hat{\alpha}$ & 0.1257 & 0.1186 & 0.1250 & 0.1068 & 0.1710 & 0.1508 \\
\hline & $\hat{\gamma}$ & 0.8001 & -0.3473 & -0.4029 & -0.5620 & -0.4867 & -0.6433 \\
\hline & $\hat{\delta}$ & 0.7272 & -0.2710 & -0.2959 & -0.4352 & -0.3585 & -0.4978 \\
\hline \multirow[t]{3}{*}{ Var } & $\hat{\alpha}$ & 0.0726 & 0.0518 & 0.0382 & 0.0367 & 0.0448 & 0.0426 \\
\hline & $\hat{\gamma}$ & 0.0678 & 0.5133 & 0.1726 & 0.1615 & 0.1818 & 0.1705 \\
\hline & $\hat{\delta}$ & 0.0943 & 0.3053 & 0.1105 & 0.1044 & 0.1151 & 0.1087 \\
\hline \multirow[t]{3}{*}{ MSE } & $\hat{\alpha}$ & 0.0883 & 0.0658 & 0.0538 & 0.0481 & 0.0741 & 0.0654 \\
\hline & $\hat{\gamma}$ & 0.7081 & 0.6340 & 0.3349 & 0.4773 & 0.4187 & 0.5843 \\
\hline & $\hat{\delta}$ & 0.6232 & 0.3787 & 0.1981 & 0.2938 & 0.2436 & 0.3565 \\
\hline $\mathrm{n}$ & \multicolumn{7}{|c|}{100} \\
\hline \multirow[t]{3}{*}{ Estimate } & $\hat{\alpha}$ & 1.5566 & 1.5752 & 1.5901 & 1.5785 & 1.6145 & 1.6018 \\
\hline & $\hat{\gamma}$ & 3.5472 & 2.4733 & 2.4522 & 2.3071 & 2.3862 & 2.2436 \\
\hline & $\hat{\delta}$ & 2.3677 & 1.4245 & 1.4198 & 1.2945 & 1.3707 & 1.2470 \\
\hline \multirow[t]{3}{*}{ Bias } & $\hat{\alpha}$ & 0.0566 & 0.0752 & 0.0901 & 0.0785 & 0.1145 & 0.1018 \\
\hline & $\hat{\gamma}$ & 0.8472 & -0.2267 & -0.2478 & -0.3929 & -0.3138 & -0.4564 \\
\hline & $\hat{\delta}$ & 0.7677 & -0.1755 & -0.1802 & -0.3055 & -0.2293 & -0.3530 \\
\hline \multirow[t]{3}{*}{ Var } & $\hat{\alpha}$ & 0.0467 & 0.0309 & 0.0228 & 0.0221 & 0.0252 & 0.0242 \\
\hline & $\hat{\gamma}$ & 0.0486 & 0.3681 & 0.2201 & 0.2051 & 0.2226 & 0.2076 \\
\hline & $\hat{\delta}$ & 0.0557 & 0.2222 & 0.1290 & 0.1211 & 0.1307 & 0.1229 \\
\hline \multirow[t]{3}{*}{ MSE } & $\hat{\alpha}$ & 0.0499 & 0.0365 & 0.0309 & 0.0282 & 0.0383 & 0.0345 \\
\hline & $\hat{\gamma}$ & 0.7664 & 0.4195 & 0.2815 & 0.3595 & 0.3211 & 0.4159 \\
\hline & $\hat{\delta}$ & 0.6451 & 0.2531 & 0.1615 & 0.2144 & 0.1833 & 0.2475 \\
\hline
\end{tabular}

It is observed from Table 3 that: the MSE of $\hat{\alpha}, \hat{\gamma}$ and $\hat{\delta}$ are decreasing when $\mathrm{n}$ is increasing using the ML and Bayesian methods. The MSE of $\hat{\alpha}, \hat{\gamma}$ and $\hat{\delta}$ are increasing when $\mathrm{n}$ is increasing using Mmethod. The best estimation method is B1sq, when the prior distribution of the parameters are gamma distribution, under squared error loss function, since the MSE of the estimators are approximately the smallest between all estimation methods. Bayes estimators under case 1, when the prior distribution of the parameters are gamma distribution have smaller MSE's than case 2, when $\alpha$ follows exponential distribution and $\gamma, \delta$ follow gamma distribution. Bayesian estimators under squared error loss function is better than Bayesian estimators under absolute error loss function.

\subsection{Application}

In this subsection, a real data set is used to compare between Weibull and $\mathrm{CW}$ distributions. [8] assumed that, the lifetime of breakdown voltages of electrical cable insulation is follow Weibull distribution. She described data at 34 kilovolts and the length of time (in minutes) until breakdown was recorded. The data set with $\mathrm{n}=19$ is: $0.19,0.78,0.96,1.31,2.78,3.16,4.15,4.67,4.85,6.50,7.35,8.01,8.27,12.06,31.75,32.52$, $33.91,36.71,72.89$. In this study, the same data is used to compare between Weibull and CW distributions. The parameters are estimated using ML method. Kolmogorov-Smirnov (K-S) test statistic for the goodness of fit, the 
Akaike information criterion (AIC), Bayesian information criterion (BIC) and the consistent Akaike information criterion (CAIC) are calculated to compare between the two distributions. The results are shown in Table 4.

Table 4: K-S, AIC, BIC and CAIC of the Weibull and CW distributions

\begin{tabular}{|c|c|c|c|c|}
\hline Model & K-S & AIC & BIC & CAIC \\
\hline Weibull distribution & 0.279 & 149.176 & 151.065 & 153.065 \\
\hline Compound Weibull distribution & 0.104 & 143.059 & 145.893 & 148.893 \\
\hline
\end{tabular}

From Table 4, we observe that the CW distribution has the lowest K-S test statistic, AIC, BIC and CAIC values compared with Weibull distribution. In this regard, the $\mathrm{CW}$ distribution provides better fit for this data set than the Weibull distribution. Thus, we may conclude that, the $\mathrm{CW}$ distribution could fit the lifetime data better than the Weibull distribution.

\section{Conclusion}

In this paper, properties of the $\mathrm{CW}$ distribution are studied with graphical representations. The HRF shape of the distribution decreasing at $\alpha<1$, approximately constant at $\alpha=1$ and unimodal at $\alpha>1$ which allows this distribution to fit different types of lifetime data. Special cases and related distributions to the CW distribution are derived. Different estimation methods are used. We find that the B1sq is the best estimation method to estimate the unknown parameters of the $\mathrm{CW}$ distribution. Bayesian estimators under squared error loss function is better than Bayesian estimators under absolute error loss function. An application on electrical cable insulation give a reliable evidence that the $\mathrm{CW}$ distribution provides a better fit than Weibull distribution.

\section{References}

[1] Dubey, S.D., A compound Weibull distribution, Naval Research Logistics Quarterly, 15,1968, 179-188.

[2] Gottschalk, L., Tallaksen, L.M., Perzyna, G., Derivation of low flow distribution functions using recession curves, Journal of hydrology, 194, 1997, 239-262.

[3] Qutb, N.S., Estimation of the Parameters of Compound Distributions, Ph.D. dissertation, Al-Azhar University, Egypt,2002.

[4] Mahmoud, M.A., Moshref, M., Yhiea, N.M., Mohamed, N.M., Progressively Censored Data from The Weibull Gamma Distribution Moments and Estimation, Journal of Statistics Applications \& Probability, 3,2014, 45-60.

[5] Fisz, M., Probability theory and mathematical statistics (New York: John Wiley and sons, 1963).

[6] Rashid, A., Jan, T., A Compound of Zero Truncated Generalized Negative Binomial Distribution With Generalized Beta Distribution, Journal of reliability and statistical studies, 6,2013, 11-19.

[7] Rinne, H., The Weibull distribution a handbook (United States: CRC Press, 2008).

[8] Lawless, J.F., Statistical Models and Methods for Lifetime Data(New York: John Wiley and Sons,2003). 